Proceedings of the ASME 2009 Turbo Expo 2020 \& Turbomachinery Technical Conference and Exposition

June 22-26, London, England, 2020, London, England

GT2020-3304

\title{
NOVEL CYCLO-NON-STATIONARY INDICATORS FOR MONITORING OF ROTATING MACHINERY OPERATING UNDER SPEED AND LOAD VARYING CONDITIONS
}

\author{
Alexandre Mauricio \\ Department of Mechanical Engineering \\ KU Leuven \\ Dynamics of Mechanical \\ and Mechatronic Systems, \\ Flanders Make \\ Celestijnenlaan 300, BOX 2420, 3001, \\ Leuven, Belgium \\ Email: alex.ricardomauricio@kuleuven.be
}

\author{
Dustin Helm \\ Bharti School of Engineering \\ Laurentian University \\ F-215C, Science Building \\ Sudbury, Canada \\ Email: dm_helm@Isbu.ac.uk
}

\author{
Markus Timusk \\ Bharti School of Engineering \\ Laurentian University \\ F-215C, Science Building \\ Sudbury, Canada \\ Email: mtimusk@laurentian.ca
}

\begin{abstract}
Jerome Antoni
Laboratoire Vibrations Acoustique University of Lyon, INSA-Lyon 69621, Villeurbanne, France

Email: jerome.antoni@insa-lyon.fr
\end{abstract}

\author{
Konstantinos Gryllias * \\ Department of Mechanical Engineering \\ KU Leuven \\ Dynamics of Mechanical \\ and Mechatronic Systems, \\ Flanders Make \\ Celestijnenlaan 300, BOX 2420, 3001, \\ Leuven, Belgium \\ Email: konstantinos.gryllias@kuleuven.be
}

\begin{abstract}
Condition monitoring arises as a valuable industrial process in order to assess the health of rotating machinery, providing early and accurate warning of potential failures and allowing for the planning and effective realization of preventative maintenance actions. Nowadays machinery (gas turbines, wind turbines etc.) manufacturers adopt new business models, providing not only the equipment itself but additionally taking on responsibilities of condition monitoring, by embedding sensors and health monitoring systems within each unit and prompting
\end{abstract}

maintenance actions when necessary. Among others, rolling element bearings are one of the most critical components in rotating machinery. In complex machines the failure indications of an early bearing damage are weak compared to other sources of excitations (e.g. gears, shafts, rotors etc.). Vibration analysis is most widely used and various methods have been proposed, including analysis in the time and frequency domain. In a number of applications, changes in the operating conditions (speed/load) influence the vibration sources and change the frequency and amplitude characteristics of the vibroacoustic signature, making them nonstationary. Under changing environments, where 
speed and load vary, the assumption of quasi-stationary is not appropriate and as a result a number of time-frequency and timeorder representations have been introduced, such as the Short Time Fourier Transform and the Wavelets. Recently an emerging interest has been focused on modelling rotating machinery signals as cyclostationary, which is a particular class of nonstationary stochastic processes. The classical cyclostationary tools, such as the Cyclic Spectral Correlation Density (CSCD) and the Cyclic Modulation Spectrum (CMS), can be used in order to extract interesting information about the cyclic behavior of cyclostationary signals, only under the assumption that the speed of machinery is constant or nearly constant. Global diagnostic indicators have been proposed as a measure of cyclostationarity under steady operating conditions. In order to overcome this limitation a generalization of both SCD and CMS functions have been proposed displaying cyclic Order versus Frequency as well as diagnostic indicators of cyclo-non-stationarity in order to cover the speed varying operating conditions. The scope of this paper is to propose a novel approach for the analysis of cyclonon-stationary signals based on the generalization of indicators of cyclo-non-stationarity in order to cover the simultaneous and independently varying speed and load operating conditions. The effectiveness of the approach is evaluated on simulated and real signals captured on a dedicated test rig.

\section{NOMENCLATURE}

\begin{tabular}{ll} 
BPFI & Ball Pass Frequency of Inner race. \\
BPFO & Ball Pass Frequency of Outer race. \\
CNS & Cyclo-Non-Stationarity. \\
ICNS & Indicators of Cyclo-Non-Stationarity. \\
WAF & Windowed Activation Function. \\
$\alpha$ & Cyclic frequency. \\
$\tau$ & Time lag. \\
$\theta$ & Angle. \\
$\Theta$ & Angle period. \\
$b w$ & Bandwidth. \\
$c f$ & Center frequency. \\
$f$ & Spectral frequency. \\
$f b$ & Frequency range of background noise. \\
$L$ & Load. \\
$p$ & Periodic signal. \\
$t$ & Time. \\
$T$ & Time period. \\
$x$ & Signal. \\
$w$ & Speed. \\
$W_{1}$ & Activation Function function of speed \\
$W_{2}$ & Activation Function function of load \\
& \\
\hline
\end{tabular}

\section{INTRODUCTION}

Condition monitoring assesses the operational health of rotating machinery, in order to provide early and accurate warning of potential failure such that preventative maintenance action may be taken. To achieve this target, manufacturers start taking on the responsibilities of engine condition monitoring, by embedding health monitoring systems within each engine unit and prompting maintenance actions when necessary.

Several types of condition monitoring are used including oil debris monitoring, temperature monitoring and vibration monitoring. Among them, vibration monitoring is the most widely used technique. Machine vibro-acoustic signatures contain pivotal information about its state of health. The current work focuses on one part of the diagnosis stage of condition monitoring for engine bearing health monitoring as bearings are critical components in rotating machinery. A plethora of signal processing tools and methods applied in the time domain [1], the frequency domain, the time-frequency domain and the time-scale domain have been presented in order to extract valuable information by proposing different diagnostic features [2]. Among others, an emerging interest has been reported on modelling rotating machinery signals as cyclostationary [3], which is a particular class of non-stationary stochastic processes. A process $x(t)$ is said to be $n^{t h}$-order cyclostationary with period $T$ if its $n^{\text {th }}$-order moments exist and are periodic with period $T$. Several tools, such as the Spectral Correlation Density (SCD) and the Cyclic Modulation Spectrum (CMS) [4] can be applied in order to extract interesting information concerning the cyclic behavior of cyclostationary signals.

In order to measure the cyclostationarity from order 1 to 4 [5], concise and global indicators have been proposed. However, in a number of applications, such as aircraft engines and wind turbines, the characteristic vibroacoustic signatures of rotating machinery depends on the operating conditions of rotational speed and/or load. During the last decades fault diagnostics of rotating machinery under variable speed/load has attracted a lot of interest [6]. In order to use the usual cyclostationary tools the speed of the machine should be assumed constant or nearly constant, otherwise the signal becomes cyclo-non-stationary. In order to overcome this limitation a generalization of both SCD and CMS functions have been proposed displaying cyclic Order versus Frequency [4].

The goal of this paper is to propose a novel approach for the analysis of cyclo-nonstationary signals based on the generalization of indicators of cyclostationarity in order to cover both speed varying and load varying conditions. The proposed indicators of cyclo-non-stationarity (ICNS) are expected to summarize the information at various statistical orders and at lower computational cost compared to the Order-Frequency SCD or CMS. The ICNS have been proposed previously to cover speed varying conditions, but the load varying conditions were not taken into account [7]. Therefore in this paper the ICNS are extended to 
cover both speed varying and load varying conditions. The rest of the paper is organised as follows: in section 2 the theory of the $n^{\text {th }}$-order cyclo-non-stationary processes is summarised and the indicators of cyclo-non-stationary are defined. In section 3 the experimental setup, used for the capturing of acceleration signals under varying operating conditions, is detailed. The novel indicators of cyclo-non-stationarity are applied on acceleration signals captured on the test rig, including a damaged bearing, operating under speed and load varying conditions and the results are discussed in section 4. Finally some first conclusions are summarised in section 5 .

\section{INDICATORS OF CYCLO-NON-STATIONARITY}

A process is termed as cyclo-stationary if its statistics are periodic. Different orders of cyclo-stationarity are defined depending on the type of statistics that is considered. A signal whose first-order statistics (i.e. the ensemble average) is periodic is said to contain first-order cyclo-stationarity. This is actually equivalent to recognizing that the signal comprises a periodic component. A signal whose second-order statistics (i.e. as returned by the instantaneous autocorrelation function) is periodic is said to contain second-order cyclo-stationarity, which reflects the existence of a periodic flow of its energy. This is typically the case of broadband processes which are periodically modulated, either in amplitude or in phase. More generally, a signal is said to contain $n^{\text {th }}$-order cyclo-stationarity if its $n^{\text {th }}$ order statistics evidences periodicity. Signals emitted by damaged bearings operating under steady conditions are usually considered as $2^{\text {nd }}$-order cyclostationary signals, due to jitter effects connected with the slippage. This definition no longer holds true if the operating conditions vary in time. Bearing signals have a time-invariant carrier connected to excited resonances of the structure, and an angle-invariant cyclic modulation connected to the impulsive cadence of the damage. The torque and the loading on the bearings also change the impact forces due to the damage. Thus, under these circumstances, bearing signals are considered as cyclo-non-stationary, as they are no longer cyclo-stationary in function of time, but retain their statistics as a function of the shaft phase and time. It is then necessary to introduce a dependence of the statistics on the machine regime. Raad et al. [5] proposed scalar indicators of $n^{\text {th }}$-order cyclosationarity, denominated as Indicators of CycloStationarity (ICS). Additionally, the authors in Ref. [7,8] extended the indicators to varying operating conditions dependent only on the speed, thus introducing the Indicators of Cyclo-Non-Stationarity (ICNS). In this paper, the dependence on the machine speed $w=d / d t$ and the machine load $L=d f / d t$ are both considered. The expected value and the autocovariance function are then defined with respect to the conditional expected values $w$ and $L$, as described in Eq. 1:

$$
m_{x}(\theta ; w ; L)=\mathbb{E}\{x(t(\theta)) \mid w, L\}=m_{x}(\theta+\Theta ; w ; L)
$$

where $\mathbb{E}\{x(t(\theta)) \mid w, L\}$ is the ensemble average of the signal $x(t(\theta))$ for a specific fixed speed value $w$ and load value $L$, and $\Theta$ is the angular period. In other words, the expected value for a specific set of load and speed is the same after one angulat period $\Theta$. This is defined as a $1^{\text {st }}$-order cyclo-non-stationary signal (CNS1) (or as the periodic part in relation to the angle $\theta$ ) when the condition of Eq. 1 is met. The $2^{\text {nd }}$-order cyclo-nonstationarity (CNS2) can similarly be defined by Eq. 2 [4]:

$C_{2 x}(\theta, \tau ; w ; L)=\mathbb{E}\left\{x_{c}(t(\theta)) x_{c}(t(\theta-\tau)) \mid w, L\right\}=C_{2 x}(\theta+\Theta, \tau ; w ; L)$

where $\tau$ is the time lag (still expressed in the time domain), with $x_{c}(t(\theta))=x(t(\theta))-m_{x}(\theta, w(\theta), L(\theta))$ being the $1^{s t}$-order part removed from the angular domain signal.

Based on Eq. 2, a process to be $2^{\text {nd }}$-order CNS is proven as a periodic function of $\theta$ function of fixed values of $w$ and $L$, if it meets the condition $C_{2 x}(\theta, 0 ; w ; L)=\mathbb{E}\left\{x_{c}(t(\theta))^{2} \mid w, L\right\}$.

Generalizing to order three, a sufficient condition for a process to be $3^{r d}$-order CNS is that $C_{3 x}(\theta, 0 ; w ; L)=$ $\mathbb{E}\left\{x_{c}(t(\theta))^{3} \mid w, L\right\}$ is a periodic function of $\theta$ function of fixed values of $w$ and $L$.

Order four requires a special treatment. Using the concept of cumulants, it can be shown that a sufficient condition for a process to be $4^{\text {th }}$-order CNS is that $C_{4 x}(\theta, 0 ; w ; L)=$ $\mathbb{E}\left\{x_{c}(t(\theta))^{4} \mid w, L\right\}-3 C_{2 x}(\theta, 0 ; w ; L)^{2}$ is a periodic function of $\theta$ function of fixed values of $w$ and $L$.

Since $C_{n x}(\theta, 0 ; w ; L), n=1,2,3,4$ is a periodic function of $\theta$, it accepts a Fourier series with Fourier coefficients $C_{n x}^{k}$, where $k$ is related to the harmonic index. Therefore, an indicator of $n^{\text {th }}$-order CNS, $n=1,2,3,4$ is returned by:

$$
\operatorname{ICNS}_{n x}(w ; L)=\sum_{k} \frac{\left|C_{n x}^{k}(w, L)\right|^{2}}{\left|C_{n x}^{0}(w, L)\right|^{n}}
$$

Without a loss of generality, let us consider the $1^{s t}$-order CNS signal observed on the time interval $[0 ; T]$,

$$
x(t)=\sum_{k} c_{x}^{k}(w, L) e^{j \alpha \theta(t)}+n(t)=p(t)+n(t)
$$

with speed-dependant and load-dependant Fourier coefficients $c_{x}^{k}(w, L)$ and additive noise $n(t)$. An estimate of $c_{x}^{k}(w, L)$ is obtained as follows. Let us first divide the speed range of interest $\left[w_{\min } ; w_{\max }\right]$ into $\mathrm{N}$ subintervals and the load range of interest $\left[L_{\min } ; L_{\max }\right]$ into M subintervals:

$$
w_{i}=w_{\min }+\Delta w, \quad i=0, \ldots, N
$$

$$
L_{j}=L_{\min }+\Delta L, \quad i=0, \ldots, M
$$


where $\Delta w=\left(w_{\max }-w_{\min }\right) / N$, and $\Delta L=\left(L_{\max }-L_{\min }\right) / M$. Let us introduce a radial basis function connected to the speed variable $w, W_{1}( \pm \infty)=0 \leq W_{1}(x) \leq W_{1}(0)=1$, centered on $x=0$, such that $W_{1}\left(w-w_{i}\right)$ returns a unit value $\left(W_{1}=1\right)$ when $w=w_{i}$ and steadily decreases to zero when $w$ gets away from $w_{i}$. Let us now create a radial basis function connected to the load variable $L, W_{2}( \pm \infty)=0 \leq W_{2}(x) \leq W_{2}(0)=1$, centered on $x=0$, such that $W_{2}\left(L-L_{j}\right)$ returns a unit value $\left(W_{2}=1\right)$ when $L=L_{j}$ and steadily decreases to zero when $L$ gets away from $L_{j}$. Then, it can be shown that:

$\widehat{c}_{x}^{k}\left(w_{i}, L j\right)=\frac{\int_{0}^{T} W_{1}\left(w(t)-w_{i}\right) W_{2}\left(L(t)-L_{j}\right) x(t) e^{-j \alpha_{k} \theta(t)} w(t) L(t)}{\int_{0}^{T} W_{1}\left(w(t)-w_{i}\right) W_{2}(L(t) w(t) L(t)}$

is a consistent estimator of the Fourier coefficient $c_{x}^{\alpha}(w, L)$ in the sense of mean square convergence as $T \rightarrow \infty$.

In turn, the signal $p(t)$ in Eq. 4 can be estimated as:

$$
\widehat{p}(t)=\sum_{k} \sum_{i=0}^{N} \sum_{j=0}^{M} W_{1}\left(w-w_{i}\right) W_{2}\left(L-L_{j}\right) \widehat{c}_{x}^{k}\left(w_{i}, L j\right) e^{-j \alpha_{k} \theta(t)}
$$

provided that the "partition of unity" condition of Eq. 9 is met.

$$
\sum_{i=0}^{N} \sum_{j=0}^{M} W_{1}\left(w-w_{i}\right) W_{2}\left(L-L_{j}\right)=1
$$

For simplification, $W_{1}$ and $W_{2}$ can be seen as Windowed Activation Functions (WAFs) to be applied on the signal $x(t(\theta))$ for speed $w_{i}$ and load $L_{i}$ respectively, and the combination of the two results lead to the general Windowed Activation Function (gWAF). The WAFs follow the generalized-Gaussian basis function :

$$
\begin{aligned}
& W_{1}\left(w-w_{i}\right)=e^{\left(\frac{\left|w-w_{i}\right|}{\beta_{1}}\right)^{2 p}} \\
& W_{2}\left(L-L_{j}\right)=e^{\left(\frac{\left|L-L_{j}\right|}{\beta_{2}}\right)^{2 p}}
\end{aligned}
$$

with $\beta_{1}=\Delta w / 2(\log (2))^{2 p}$ and $\beta_{2}=\Delta L / 2(\log (2))^{2 p}$, and $p=$ 1.706. The multiplication of all combinations of $W_{1}$ and $W_{2}$ results in the activation functions gWAF for each combination of specific speed with specific load.

The extraction of the ICNS can be synthesized in the next steps:

Step 1: First, the raw signal $x(t)$ is resampled in the angular domain $x(t(\theta))$ based on the speed signal.
Step 2: The resolution of each discrete speed $\Delta w$ and load $\Delta L$ are selected and the speed samples $w_{i}$ and load samples $L_{j}$ are extracted.

Step 3: The Windowed Activation Function (WAF) for one speed $w$ and one load $L$ are calculated based on Eq.10 and Eq. 11 to calculate $W_{1}\left(w-w_{i}\right)$ and $L_{1}\left(L-L_{j}\right)$.

Step 4: The $W_{1}$ and $W_{2}$ are multiplied to each other and applied on the signal $x(t(\theta))$ to obtain the part corresponding to the specific speed and load.

Step 5: Calculate the periodic part of the signal $\widehat{p}(t)$. This can be done through synchronous averaging, as was initially suggested in Ref. [9], or through other methods such as the Linear Prediction and the Cepstrum Editing.

Step 6: Repeat steps 2 through 5 for all the other combinations of speed and load $(w, L)$.

Step 7: Sum all periodic parts $\widehat{p}(t)$ to obtain the full periodic signal and subtract it from the original signal $x(t(\theta))$ to obtain the residual signal $x_{c}(t(\theta))$.

Step 8: Obtain the $n^{\text {th }}$-order CNS signal by calculating the square of the residual signal $\left(x_{c}^{2}\right)$, the residual signal to the power of $3\left(x_{c}^{3}\right)$, and the residual signal to the power of 4 minus 3 times the $2^{\text {nd }}$-order CNS signal $\left(x_{c}^{4}-3 x_{c}^{2}\right)$.

Step 9: Apply the already calculated gWAF for each combination of speed and load to obtain the signals as function of speed and load for eack $n^{\text {th }}$-order $\left(C_{n x}^{k}(w, L)\right)$.

Step 10: Apply the normalization described by Eq. 3, by calculating the mean of the $C N S_{n x}$ signal of the $n^{\text {th }}$-order and its square, in order to obtain the $\operatorname{ICN} S_{n x}(w ; L)$.

The ICNS should prove to be higher when bearing damages are present, because it is expected that a faulty rolling element bearing will exhibit an increase of ICNS corresponding to the incriminated fault frequency, in particular:

on order $n=1$ when the fault frequency and its first harmonic occur in a band of high signal-to-noise ratio.

on order $n=2$ when the fault is carried by components so high in frequency that they are nearly random in nature.

on order $n=3$ due to the impulsive nature of bearing faults in their early stage (localized defect).

at a set combination of speed and load which are critical in nature for the rotating structure and more favourable to the appearance of the bearing faults.

\section{EXPERIMENTAL SETUP}

The proposed indicators of CNS are tested in a parallel drive system depicted in Fig. 1 under varying operating conditions of speed and load.

The system is driven by a 10 HP AC electric motor. Power is transmitted through a reduction gear box with a ratio of $3: 1$ to the drive pulley of the belt drive system. The load is applied to the pump pulley by a hydraulic gear pump connected to a solenoid 


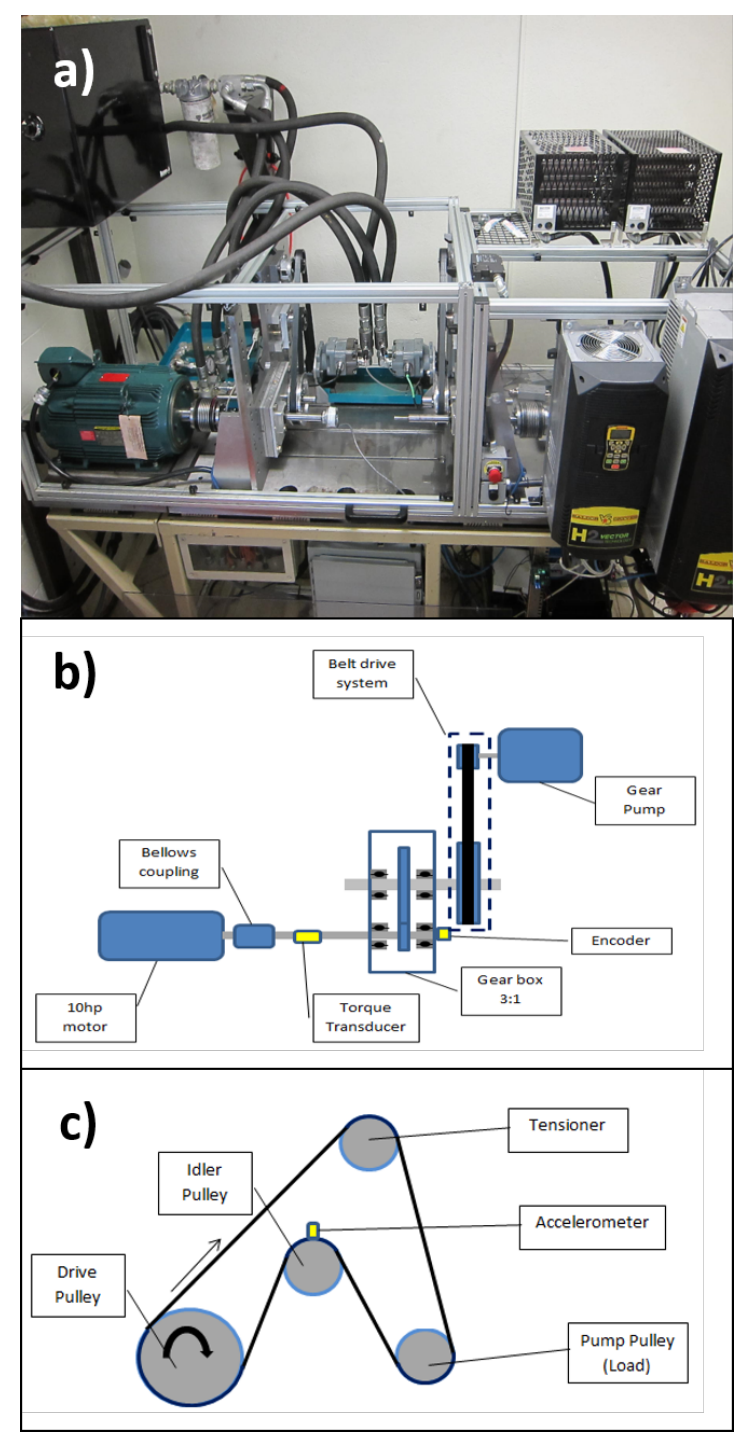

FIGURE 1: (A) PHOTO OF THE TEST TIG, (B) SCHEMATIC OF THE TEST RIG, AND (C) SCHEMATIC OF THE BELT DRIVE SYSTEM.

pressure controlled valve used to regulate the load, which is measured in pressure (bar). The studied damage is introduced on the inner race or on the outer race of a SKF 6203 bearing in the idler pulley. The idler pulley diameter is $76 \mathrm{~mm}$ and the drive pulley diameter is $160.3 \mathrm{~mm}$, resulting in a speed increase of 1:2.11. The full ratio between the motor and the outer rotating race of the test bearing is then equal to $1.42: 1$.

The characteristic orders of the bearing can be calculated based on the geometry: the Ball Pass Order of the Outer race (BPOO) is equal to 2.14 orders of the motor speed; and the Ball Pass Order of the Inner Race (BPOI) is equal to 3.47 orders of the a)

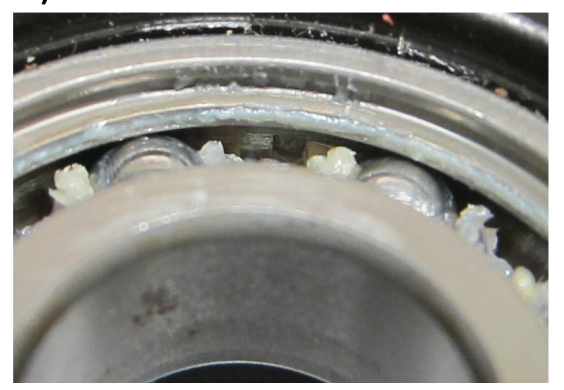

b)

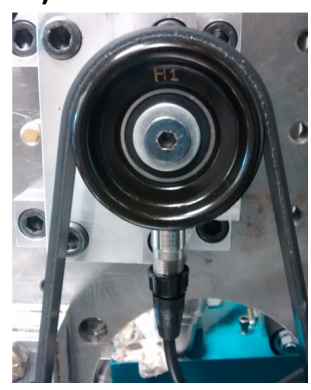

FIGURE 2: (A) BEARING WITH OUTER RACE DAMAGE OF 1.5 MM, (B) IDLER PULLEY.

motor speed. The test has been performed for three conditions: healthy; outer race damage of $1.5 \mathrm{~mm}$ width; inner race damage of $1.5 \mathrm{~mm}$ width. The accelerometer was mounted on top of the idler pulley where the test bearing is mounted. Figure 2 shows a close up on the bearing with the outer race damage and the idler pulley.

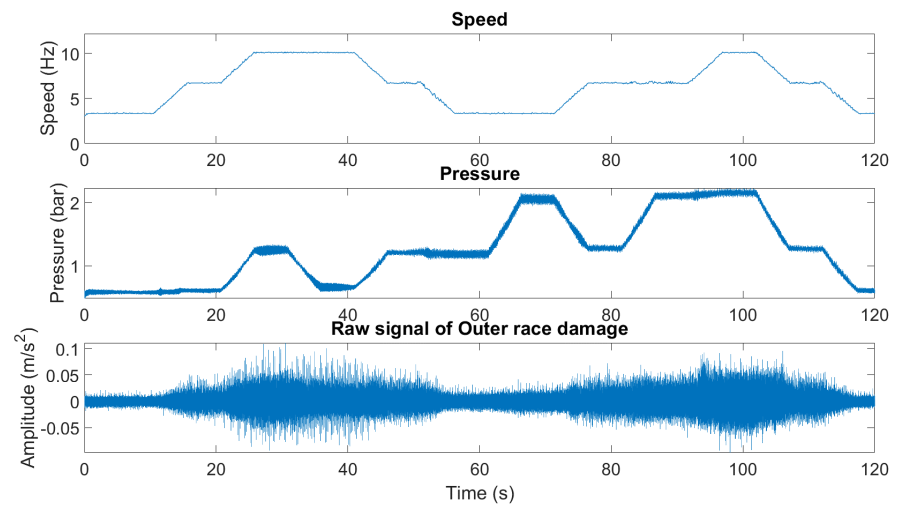

FIGURE 3: ACQUIRED SIGNAL OF SPEED, PRESSURE AND ACCELERATION OF OUTER RACE DAMAGE CASE.

A Barksdale 423X with a maximum range of 3000 psi was used to capture the load signal with a sampling frequency of 10 kHz. Similarly, a PCB Piezoelectronics 603C01 accelerometer was used to capture the vibration signals at a sampling frequency of $10 \mathrm{kHz}$. A dual channel incremental optical encoder BEI XHS25 was used to record and control the rotational speed of the motor,with a sampling frequency equal to $10 \mathrm{~Hz}$. The data was acquired by a National Instrument CompactRIO system connected to a computer with NI LabVIEW software for the synchronization of the acquired signals and the control of the pressure and of the speed. The signals used in this paper were 
a)
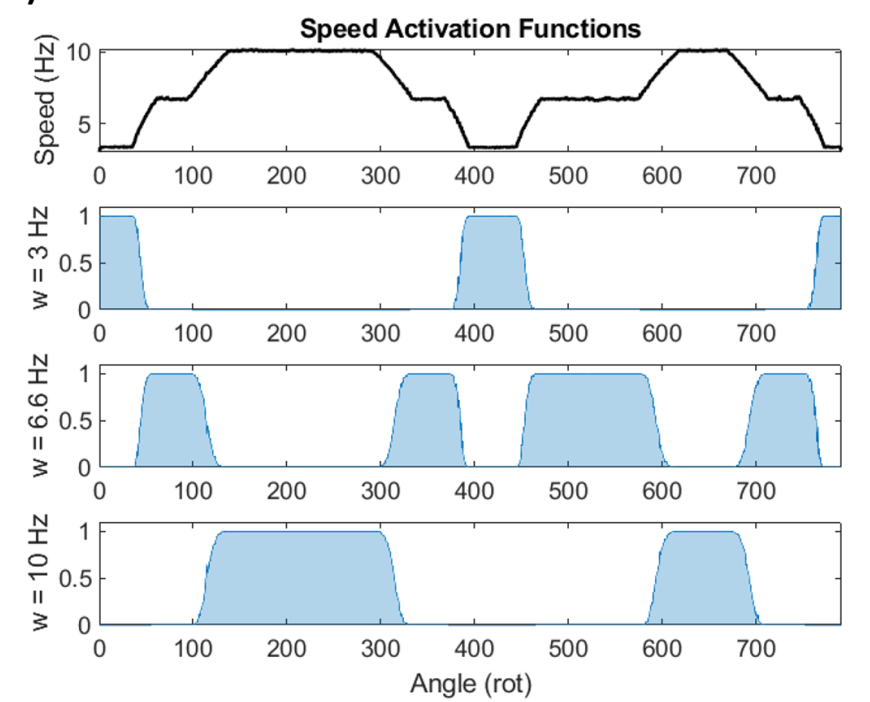

b)

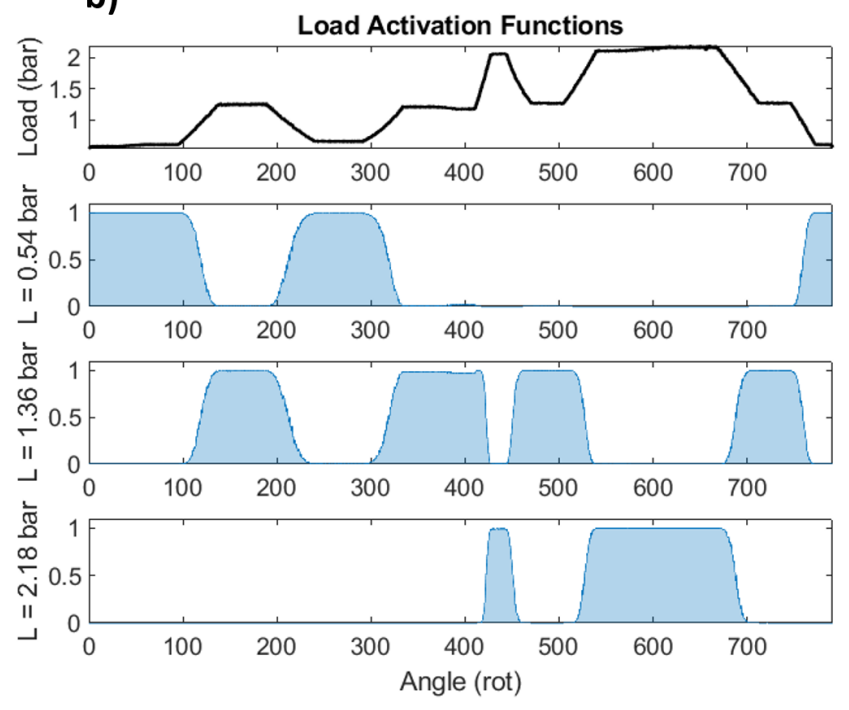

FIGURE 4: (A) ACTIVATION FUNCTIONS FOR SPEED, (B) ACTIVATION FUNCTIONS FOR LOAD.

captured under varying speed conditions ranging from $3.3 \mathrm{~Hz}$ to $10 \mathrm{~Hz}$, following the speed profile shown in Fig. 3.

The load applied on the system can also been seen in the pressure profile of Fig. 3. Signals from the other two cases (healthy and inner race damage) were captured under the same speed and loading conditions. The acceleration signal of the outer race case is demonstrated in the Fig. 3.

\section{EXTRACTION OF ACTIVATION FUNCTION $W_{i, j}$}

The following examples illustrate the activation functions for the speed-dependant radial basis function $W_{1}(w)$ and for the load-dependant radial basis functions $W_{2}(L)$. The minimum considered speed is $w_{\min }=3.3 \mathrm{~Hz}$ and the maximum speed is $w_{\max }=10 \mathrm{~Hz}$. The resolution is defined as $\Delta w=3.3 \mathrm{~Hz}$, resulting in 3 speed samples $w_{i}=[3.3 ; 6.6 ; 10]$. Analysing Fig. 4 it can be demonstrated that the speed-dependant WAF $W_{1}$ captures well the instances where the signal operates at each specific speed .

The same can also be observed from the load-dependant WAF $W_{2}$ for the 3 load samples $L_{i}=[0.54 ; 1.36 ; 2.18]$ by analysis of each WAF compared with the load profile demonstrated also on Fig. 4.

The final activation function $W_{i, j}$, which is both speed and load dependant, is the combination of the 3 discrete speeds with the 3 discrete loads, resulting in a total of 9 activation functions shown in Fig. 5.

The example demonstrated in this section corresponds to the outer race damaged case, but both other cases (healthy and inner race damage) operated under the same load and speed condition, following the same profiles. Thus, all cases resulted on the same activation functions.

\section{RESULTS OF ICNS}

Following the proposed methodology, the measured speed signals are used to perform angular resampling of the raw signal. The speed is seen to be a good approximation of the real speed, however the resolution is seen to be too low for extraction of the periodic part $\widehat{p}(t)$ by the Generalized Synchronous Averaging (GSA), proposed in [4]. At the highest speed operating condition of $10 \mathrm{~Hz}$, the resolution is $0.167 \mathrm{~Hz}$, which can lead up to $1.67 \%$ error in the speed estimation. The implemented GSA resulted in a periodic signal $\widehat{p}(t)$ with very low amplitude, equal to $1 / 100$ of the full signal. The authors then decided to use the Linear Prediction (LP) based on the AutoRegressive (AR) model to extract the periodic part $[10,11]$.

As mentioned above, the speed and the load were decomposed into $N=3$ intervals, resulting in nine activation functions and nine signals. Next, the periodic part $\widehat{p}(t)$ was calculated with an AR model of order defined by the Akaike Information Criterion (AIC) [12], and the periodic part was removed to obtain the residual signal $\widehat{x}_{c}(t)=x(t)-\widehat{p}(t)$. The residual signal is then filtered in the frequency band $[2.5 ; 5] \mathrm{kHz}$, that is likely to enhance the bearing damage. This was performed on an empirical basis, although more principled approaches could have been used in the selection of the band, such as the Fast Kurtogram or the IESFOgram. The ICNS were then computed for orders 2 to 4 . The indicators corresponding to the outer race damaged case are 

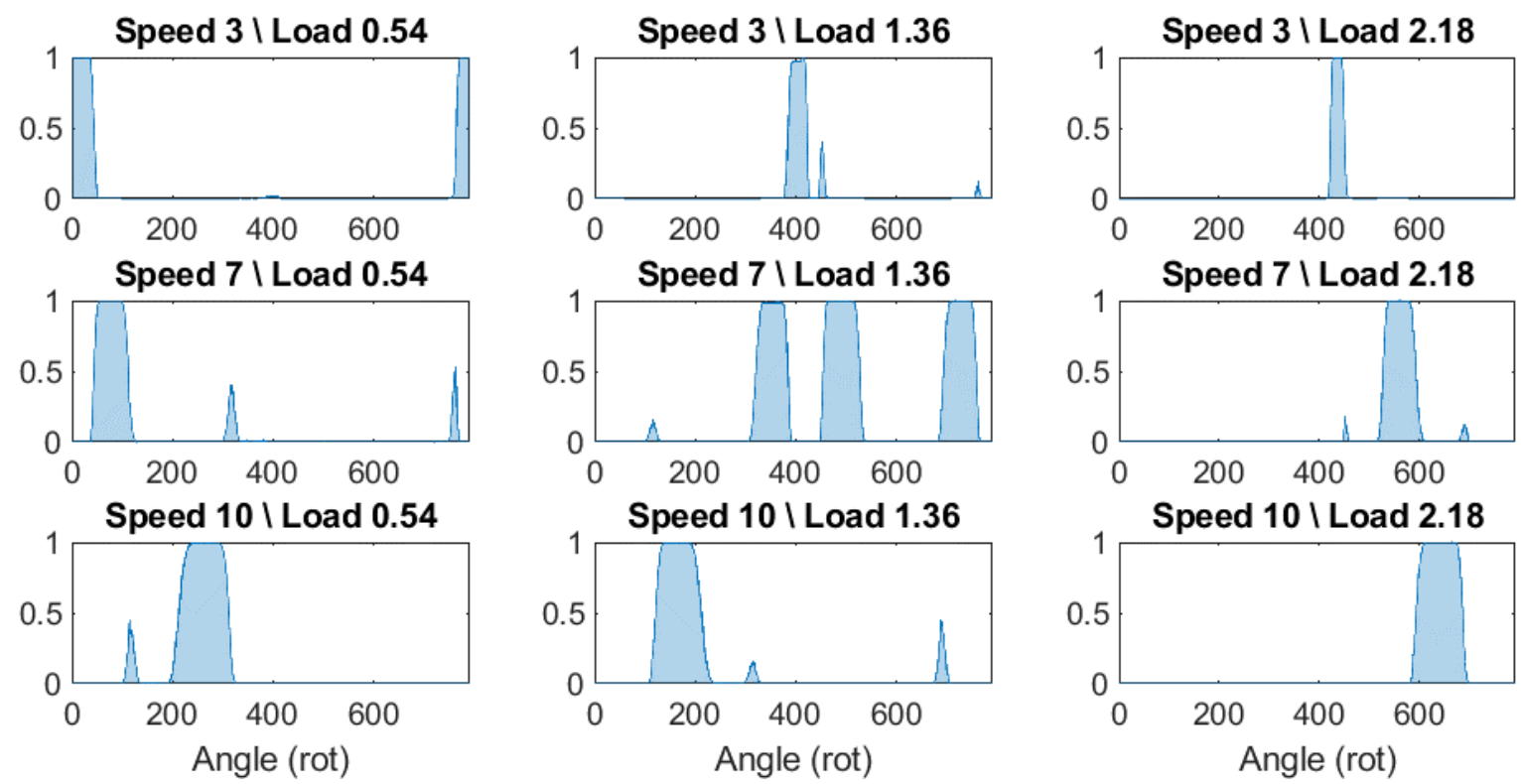

FIGURE 5: ACTIVATION FUNCTIONS FOR EACH COMBINATION OF SPEED AND LOAD.

a)
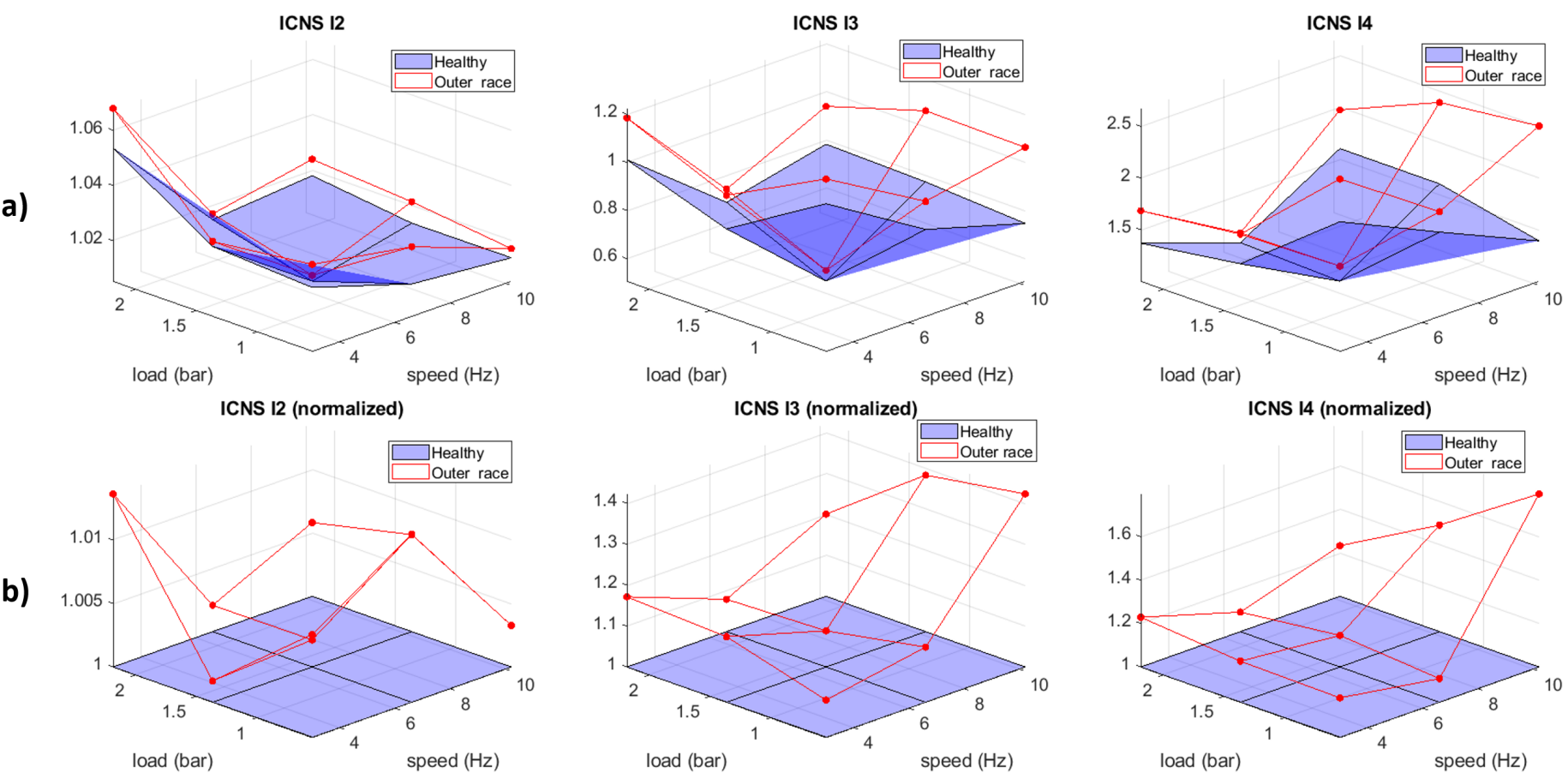

FIGURE 6: INDICATORS OF CYCLO-NON-STATIONARITY FOR THE OUTER DAMAGED CASE FOR THE $2^{\text {nd }}, 3^{\text {rd }}$ AND $4^{\text {th }}$ ORDER (A) ABSOLUTE VALUES, (B) NORMALIZED BY THE HEALTHY CASE.

presented in Figure 6. A version of the indicators normalized by the healthy case is also added to Fig. 6 for an easier visualization of the evolution of the faulty indicators in relation to the healthy benchmark. The normalized indicators are just the indicator val- 

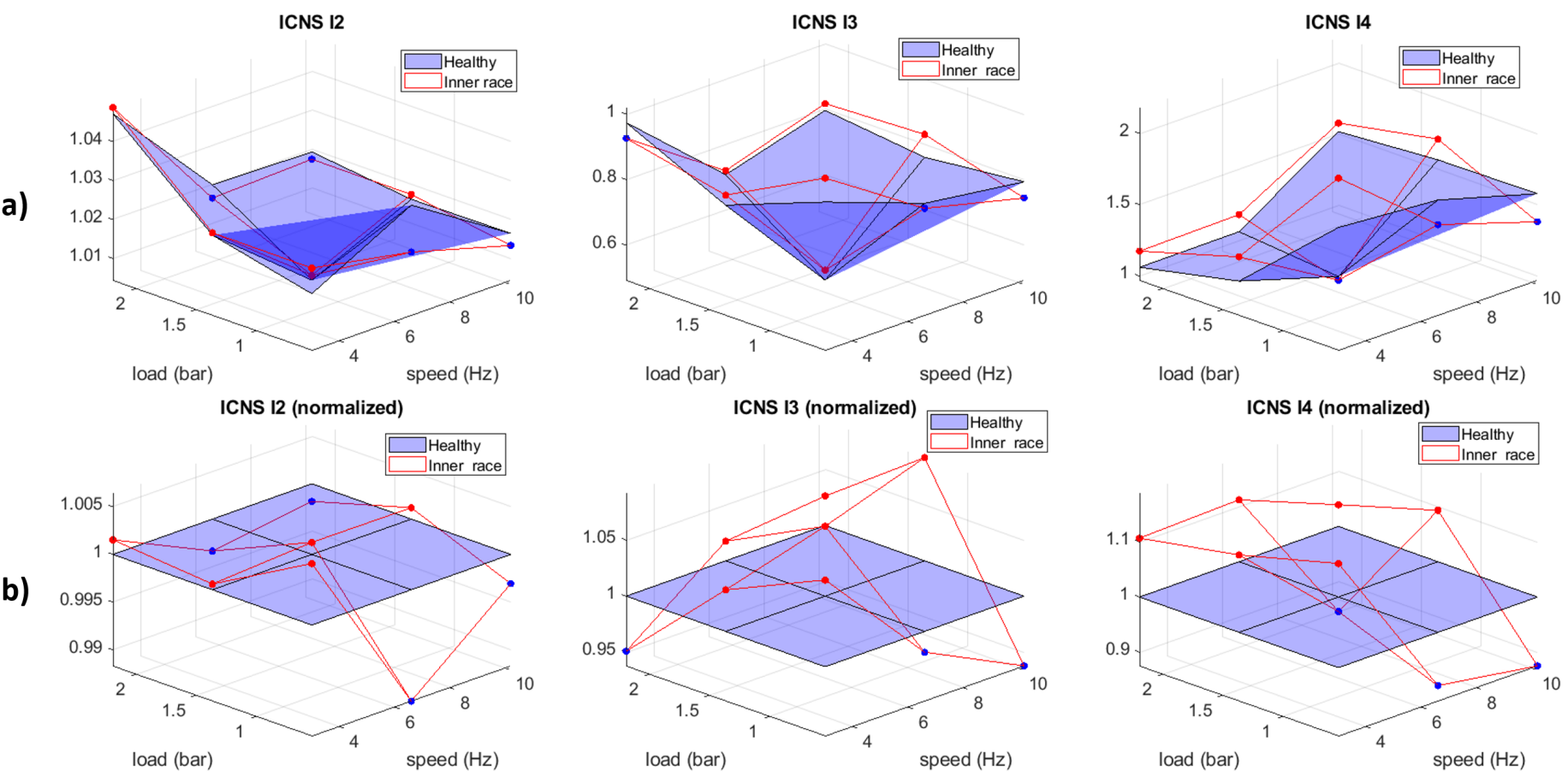

\section{FIGURE 7: INDICATORS OF CYCLO-NON-STATIONARITY FOR THE INNER DAMAGED CASE FOR THE $2^{\text {nd }}, 3^{\text {rd }}$ AND $4^{\text {th }}$ ORDER (A) ABSOLUTE VALUES, (B) NORMALIZED BY THE HEALTHY CASE.}

ues divided by the healthy indicators for each load and speed combination, thus making the normalized healthy ICNS unitary values.

It can be seen that the indicators of all orders for all speed and load cases follow the same trend. Furthermore, the indicators of the outer race damaged signals show higher values than the healthy ones. This is expected, as the periodic part removes the periodic harmonics in both healthy and damaged signals, and thus only the impulsive response of the damaged should remain on the damaged case. Another observation is that the indicators seem to not correlate with the increase of speed or load, indicating the system to be highly non-linear.

The same trend between the inner race damaged case and the healthy case can also be seen in the ICNS shown in Fig. 7. Here is can be detected for some combinations of load and speed that the ICNS present lower values than the healthy case, represented as blue dots on Fig. 7, which are considered as miss-detections. On the other hand, most of the inner race case indicators are above the healthy indicators (represented by red dots).

Unlike most common application, the inner race is stationary in this setup while the outer race is rotating. The load zone in relation to the position of the inner race damage is not clear, and at different loads and speeds it may cause the rolling element bearing to not provide a strong enough impact to be captured by the sensor, thus causing the missed detections. On the other hand, the outer race damage may mitigate this due to the fact that damage is rotating constantly through the load zone, providing no missed detections.

\section{CONCLUSION}

In this paper a novel approach for the analysis of cyclonon-stationary signals by indicators measuring the cyclo-nonstationarity of the signals in function of speed and load is proposed for applications which operate under varying speed and varying load conditions. The proposed indicators summarize the information at different statistical orders with low computational cost, especially when compared to other cyclo-non-stationary based tools. The indicators have been studied on an experimental setup for two bearing damage cases: an inner race defect; and an outer race defect. The indicators of the outer race damaged case show excellent performance in achieving higher statistical orders values than the healthy case, while the majority of speed-load combinations of the inner race damaged case also show higher values when compared to the healthy case. The proposed indicators can be concluded to be a possible methodology for bearing damage diagnosis under varying operating conditions. 


\section{REFERENCES}

[1] McFadden, P., and Smith, J., 1984. "Vibration monitoring of rolling element bearings by the high frequency resonance technique: A review". Tribology International, 117(1), pp. 3-10.

[2] Randall, R., and Antoni, J., 2011. "Rolling element bearing diagnostics - a tutorial". Mechanical Systems and Signal Processing, 25(2), pp. 485-520.

[3] Antoni, J., 2009. "Cyclostationarity by examples". Mechanical Systems and Signal Processing, 23(4), pp. 987 1036.

[4] Abboud, D., Baudin, S., Antoni, J., Remond, D., Eltabach, M., and Sauvage, O., 2016. "The spectral analysis of cyclonon-stationarity signals". Mechanical Systems and Signal Processing, 75, pp. 280-300.

[5] Raad, A., Antoni, J., and Sidahmad, M., 2008. "Indicators of cyclostationarity: Theory and application to gear fault monitoring". Mechanical Systems and Signal Processing, 22(3), pp. 574-587.

[6] Heyns, T., Godsill, S., de Villiers, J., and Heyns, P., 2012. "Statistical gear health analysis which is robust to fluctuating loads and operating speeds". Mechanical Systems and Signal Processing, 27, pp. $651-666$.

[7] Gryllias, K., Moschini, S., and Antoni, J., 2018. "Application of cyclo-nonstationary indicators for bearing monitoring under varying operating conditions". Journal of Engineering for Gas Turbines and Power, 140.

[8] Antoni, J., Abboud, D., Raad, A., and Gryllias, K., 2016. "Application of cyclo-non-stationary indicators for bearing health monitoring under varying operating conditions". In Proceedings OF ISMA2016 INCLUDING USD2016, A. Editor and B. Editor, eds.

[9] Abboud, D., Antoni, J., Sieg-Zieba, S., and Eltabach, M., 2016. "Deterministic-random separation in non-stationary regime". Journal of Sound and Vibration, 362, pp. 305326.

[10] Sawalhi, N., and Randall, R. B., 2006. "Helicopter gearbox bearing blind fault identification using a range of analysis techniques". In Engineering Asset Management, J. Mathew, J. Kennedy, L. Ma, A. Tan, and D. Anderson, eds., Springer London, pp. 724-737.

[11] Wang, W., and Wong, A. K., 2002. "Autoregressive ModelBased Gear Fault Diagnosis ”. Journal of Vibration and Acoustics, 124(2), pp. 172-179.

[12] Akaike, H., 1969. "Fitting autoregressive models for prediction". Annals of the Institute of Statistical Mathematics, 21(1), Dec, pp. 243-247. 